\title{
MULTIPLE-SUPERNOVA REMNANTS
}

\author{
M. Różyczka \\ Warsaw University Observatory \\ Al. Ujazdowskie 4, PL-00-478 Warszawa, Poland
}

\begin{abstract}
The present observational and theoretical status of multiple-supernova remnants is briefly reviewed, and evolutionary models of the remnants around $O B$ associations are presented. The remnants are followed for up to almost $30 \mathrm{Myr}$ in various galactic environments (Gaussian or composite disks combined with cold or hot halos). It is found that the presence of a hot halo profoundly modifies the evolution of the remnants, promoting the evaporation of their shells and leading to noneruptive merging of the ejecta with the halo gas. The results also suggest an explanation for the observed deficiency of evolved multiple-supernova remnants.
\end{abstract}

\section{INTRODUCTION}

Multiple-supernova remnants (hereafter MSR) is a fairly complicated subject. Both theory and observations of MSRs provide us with more controversies than ultimate conclusions, and in principle every controversy should be given its share of author's attention. As for the present status of the theory, one has to divide the available time and space between things that have been done and things that should be done, and a fair reviewer would probably do it on a fifty-fffy basis. But what makes the situation really complicated, is the multitude of proven or expected connections leading from MSRs to such issues of the galactic astronomy like circulation of chemically enriched gases, galactic winds, or structure and state of the galactic halo. With similar connections pointing towards propagating star formation, the MSRs seem to be one of the vital links in the still mysterious chain of events responsible for the chemical evolution of galaxies.

To get a MSR, one needs an $\mathrm{OB}$ association and a galactic disk. An average association contains 20-40 stars of spectral type B3 or earlier whose masses are greater than $8 \mathrm{M}_{\odot}$, and which can explode as type II supernovae (the number of stars in "record" associations can be 10-20 times larger). One-tenth of those stars are massive ( $M>$ $30 \mathrm{M}_{\odot}$ ), rapidly evolving $\mathrm{O}$ stars, which are powerful sources of ionizing radiation and stellar winds. It can be estimated, however, that more than $80 \%$ of the energy deposited by the association in the disk is provided by supernovae originating from less massive and more slowly evolving $B$ stars. The least massive supernova progenitors explode after $\sim 3 \times 10^{7} \mathrm{yr}$, during which the association expands at an average rate of $\sim 5 \mathrm{~km} / \mathrm{s}$. Thus, the size of a dying association is on the order of $150 \mathrm{pc}$. MSRs can easily grow to sizes appreciably larger than that, which means that from their point of wiev the associations can be regarded as essentially point-like sources of multiple explosions. 
Gaseous galactic disks receiving the energy liberated by OB associations are highly nonuniform, differentially rotating structures permeated by magnetic fields and enveloped in hot halos. To make the things worse, they are very likely turbulent. On the average, their density decreases rapidly as one moves away from the equatorial (or symmetry) plane. The best fits to Ly- $\alpha$ and $21 \mathrm{~cm}$ data reflecting this decrease contain Gausssian as well as exponential components. The density of a halo is thought to be on the order of 0.003 atoms $/ \mathrm{cm}^{3}$, and the temperature of a halo - on the order of $1-3 \times 10^{6} \mathrm{~K}$. The Galactic halo is loosly described as "a disk with $\sim 5 \mathrm{kpc}$ thickness (Heiles 1987).

An evolving MSR sweeps the disk gas and stores it in a cool and dense shell which in most Galactic cases is the only observationally detectable part of the whole remnant (in other galaxies the swept volume itself can be detected as a "hole" in the disk). Thus, from the observational point of view the Galactic MSRs belong to the class of largescale HI structures (among these latter, ring-like or arc-like features prevail outside of the solar circle, while in the inner Galaxy tightly packed, roughly cylindrical "worms" are almost exclusively found). In the Milky Way there is no one-to-one correspondence between $O B$ associations and radio features. On the other hand, the HI holes in M31 (especially those with diameters $\leq 300 \mathrm{pc}$ ), as well as the HI shells in Magellanic Clouds, seem to be rather well correlated with $\mathrm{OB}$ associations and HII regions (Tenorio-Tagle and Bodenheimer 1988 and references therein).

It has been suggested (Heiles 1987) that the observed volume of the Galactic disk occupied by MSRs is much smaller than the arguments based on the total number and mean lifetimes of associations would suggest (similar discrepancies have also been found in other galaxies). Moreover, the expected mass input rate to the Galactic halo due to supernovae from $O B$ associations seems to significantly exceed the one implied by the diffuse X-ray data. Coupled with the above controversies, the widespread belief that the sequential supernovae in $\mathrm{OB}$ associations dominate the dynamics and morphology of the interstellar medium caused the evolution of MSRs to become an increasingly attractive theoretical challenge.

The first suggestion that large HI structures (or at least some of them) may be identified with MSRs was due to Cowie et al. (1979). The theory of MSRs has been developed since then by Bruhweiler et al. (1980), Tomisaka et al. (1981), Tomisaka and Ikeuchi (1986), Ikeuchi (1987), McCray and Kafatos (1987), Tenorio-Tagle et al. (1987, hereafter Paper I), Mac Low and Mc Cray (1988), Mac Low et al. (1989, hereafter MLMCN) and Igumentshchev et al. (1989). The methods applied by those authors ranged from analytical estimates based on the classical theory of interstellar bubbles, through one-dimensional hydrodynamical simulations of spherically symmetric MSRs and simplified two- dimensional hydrodynamics (snowplough and Kompaneyets approximations), to full two-dimensional simulations.

In the theoretical investigations, the associations are approximated as point sources of either "superwinds" or repeating explosions. In the first scenario the mechanical luminosity of a superwind is defined as the energy of all supernovae which explode in the association divided by the lifetime of the association. Based on the initial mass function and stellar lifetimes, the time intervals $\Delta T_{\mathrm{sn}}$ between consecutive explosions 
in the second scenario can be shown to differ very little from each other (Tomisaka and Ikeuchi 1986, Mac Low and Mc Cray 1988), and the commonly adopted value of $\Delta T_{s n}$ is $2 \times 10^{5} \mathrm{yr}$. The disks are represented by smooth density distributions in hydrostatic equilibrium, stratified in the direction perpendicular to the equatorial plane. Self-gravity, magnetic fields and rotation are usually neglected (see, however, Umemura et al. 1988, and Palouš, this volume). The microphysical processes taken into account in theoretical papers on MSRs include optically thin cooling (in all cases), heat conduction (in one case) and various approximation to the evaporation from the shell (in nearly all cases).

Some theoretical results seem to be already fairly well established. Mac Low and Mc Cray (1988) found in agreement with Paper I that in an evolved MSR the blast waves from subsequent supernovae decay into sound waves before they hit the outer shell. Further, based on the Kompaneyets approximation, they formulated a criterion predicting whether an MSR would break out of a stratified gaseous disk of a galaxy. MLMCN and Igumentshchev et al. (1989), who modelled the breakout phenomenon with the help of 2-D hydrodynamical codes, found in agreement with that criterion that the breakout occurs readily in a Gaussian disk but may be entirely prevented in an exponential disk with a scale height of a few hundred parsecs.

However, even 2-D simulations have not yet led to a fully consistent theoretical description the evolution of MSRs. A good example of a disputed issue is shell instabilities. All authors agree that the shell of a MSR evolving in a stratified disk becomes appreciably irregular due to the Rayleigh-Taylor instability which begins to operate as soon as the breakout commences (i.e. as the velocity of the upper part of the shell starts to increase). However, according to our results from Paper I the shell can become irregular even without the presence of a density gradient in the ambient medium. We attributed this effect to either the Rayleigh-Taylor instability induced by supernova shocks decaying into sound waves before they hit the shell, or a more general, thin shell instability discussed e.g. by Różyczka (1985) or Bertschinger (1986). It must be stressed that the early presence of shell irregularities may significantly increase the diffusion rate through the shell/ejecta interface, and influence the MSR evolution due to strongly enhanced cooling of the interior still prior to the breakout.

Also, entirely open problems connected with MSRs exist in addition to the unsettled ones. The results of MLMCN and Igumentshchev et al. (1989), who estimated that during the breakout 5-10\% of the outer shell and a large fraction of the ejecta accelerate into the halo, indicate that the MSRs are likely to play the major rôle in the processes of mixing between galactic disks and halos, and that MSR modelling may provide important informations about the chemical evolution of spiral galaxies (see e.g. Ikeuchi 1987). However, if the exchange of matter between the disk and the halo is to be followed, the calculations should be extended into much later evolutionary phases of the MSRs than it has been done so far. Also, various gas distributions should be examined in order to clarify their influence on MSR evolution and/or circulation of supernova ejecta.

Another open problem is the very possibility of the breakout in a composite disk which constitutes the best fit to the Lyman- $\alpha$ and $21 \mathrm{~cm}$ observations of HI (Lockman et al. 1986, Bloemen 1987). According to Mac Low and Mc Cray (1988), in such a 
medium the breakout may not occur, and as a result giant, coherent $\mathrm{HI}$ arcs or shells would be formed. It can be calculated that more than 2000 structures of this type should be seen in the Galaxy, which is clearly not the case.

To look at these questions, Tenorio-Tagle, Różyczka and Bodenheimer (1989, hereafter MRB) obtained a broad sample of evolutionary models of MSRs, some of which were followed for over $17 \mathrm{Myr}$ (up to almost $30 \mathrm{Myr}$ in one of the cases). Their models evolve in various environments (Gaussian, exponential or composite disks combined with hot or warm halos), and in several cases the diffusive effects are included in a simplified way. A brief description of their numerical methods and input physics is given in Section II. Section III presents some of their models. Finally, the results of MRB and their implications are discussed in Section IV.

\section{THE CALCULATIONS}

a) Numerical methods and physical assumptions

The equations of hydrodynamics are solved on a cylindrical grid by means of an explicit, second order scheme described by Różyczka (1985). Due to symmetry constraints only one quadrant of the remnant is actually followed. The $(r, z)$ grid consists of $100 \times 200-300 \times 600$ equally spaced points and provides a resolution of $\Delta \mathrm{r}=\Delta \mathrm{z}=$ $5.0-2.5$ pc.

Radiative, optically thin cooling operates everywhere in the grid (except in the undisturbed gas, in order to keep the latter in the dynamical equilibrium during the calculations). Magnetic fields and self-gravity are neglected. The models are subject, however, to an external gravitational field defined in such a way that the gravitational acceleration balances pressure gradients in the undisturbed gas at the beginning of the evolutionary run. Only the z-component of that field is different from zero, and its value is lower than the Galactic one by factors of 2 to 50 at low and high latitudes, respectively. The deceleration of MSR shells is therefore uderestimated. However, since at the end of each run the characteristic time for the real deceleration is always longer than, or equal to the evolutionary time, the errors introduced in this way should not be too significant.

b) Initial and boundary conditions

A pulsed energy input is employed, i.e. all consecutive supernova explosions are modelled separately. Every explosion is induced by an instantaneous release of $2.5^{50}$ ergs of thermal energy within a small volume representing a quarter of a sphere centered at $\mathrm{z}=\mathrm{r}=0$. Following the estimates discussed by Tenorio-Tagle and Bodenheimer (1988), a value of $2 \times 10^{5} \mathrm{yr}$ is chosen for the interval between explosions $\left(1 \times 10^{6} \mathrm{yr}\right.$ in one of the cases). The mass of the ejecta is limited to $8 \mathrm{M}_{\odot}$ per single explosion.

In several cases the diffusive effects are simulated by adding an extra mass to the ejecta of every supernova while leaving the mass of the shell unchanged. For the extra mass a value of $50 \mathrm{M}_{\odot}$ is adopted, resulting from the formula given by Mac Low and McCray (1987) for temperatures slightly above $10^{6} \mathrm{~K}$. The total mass injected into "diffusive" MSRs amounts to about $6000 \mathrm{M}_{\odot}$, while the total mass of the evolved 
shell is one order of magnitude larger. Admittedly, this is a very crude description of the diffusion. However, since the diffusion affects the evolution of a MSR mainly by enhanced cooling of the interior, such a procedure is justified at least as a first approximation to the problem. To check its validity, in one of the "diffusive" models the mass was gradually removed from the shell: no qualitative differences in the flow pattern were observed.

In the present calculations two types of density distributions of the gaseous component of a galactic disk are considered. The scale height $H$ of the pure Gaussian disk $\rho=\rho_{0} \exp \left(-(z / H)^{2}\right)$ is equal to $100 \mathrm{pc}$, whereas in the composite disk $\rho=$ $\rho_{e} \exp \left(-\left(z / H_{e}\right)+\rho_{g} \exp \left(-\left(z / H_{g}\right)^{2}\right)\right.$ the scale heights $H_{e}$ and $H_{g}$ amount to 300 and 100 pc (Fuchs and Thielheim 1979). The parameters $\rho_{0}, \rho_{e}$ and $\rho_{g}$ are equal to $1.67 \times 10^{-24}$, $1.25 \times 10^{-24}$ and $0.42 \times 10^{-24} \mathrm{~g} / \mathrm{cm}^{3}$, respectively. In all models the pressure $P_{0}$ in the symmetry plane of the disk is close to the canonical value of $1 \times 10^{-12} \mathrm{dyn} / \mathrm{cm}^{2}$ (Bloemen 1987).

The disks are either isothermal $\left(T_{D}=3 \times 10^{3} \mathrm{~K}\right)$, or the temperature rises in them from $7.5 \times 10^{3} \mathrm{~K}$ in the symmetry plane up to several hundred thousand $\mathrm{K}$ far away from it. As a result, in nonisothermal disks the pressure gradient is much gentler than the density gradient. According to Bloemen (1987), such a situation is observed in the Galaxy when the effective (i.e. turbulent and magnetic) pressure is considered instead of the purely thermal one.

The Gaussian models and most of the composite ones have a uniform halo which extends above $280 \mathrm{pc}$ from the galactic plane and remains in pressure equilibrium with the stratified part of the disk. The density of the halo $\rho_{H}$ is in all models equal to $5 \times 10^{-27} \mathrm{~g} / \mathrm{cm}^{3}$, while its pressure $P_{H}$ ranges from $3 \times 10^{-15}$ to $7 \times 10^{-13} \mathrm{dyn} / \mathrm{cm}^{2}$, resulting in halo temperatures $T_{H}$ of $7.5 \times 10^{3}-1 \times 10^{6} \mathrm{~K}$.

\section{RESULTS OF CALCULATIONS.}

All MRB cases discussed here are listed in Table 1, in which "evp" denotes the model in which the gas was artificially removed from the shell. The remaining, selfexplanatory abbreviations refer to the information contained in Section II.

Cases A-B.

These models (Fig.1) illustrate the possibility of the breakout in composite and Gaussian disks. Case A (Fig. 1a) was run at a resolution of 3.3 pc for $2.91 \times 10^{7}$ yr. At that time several dense tongues resulting from the shell instability separate from the main shell and plunge into the MSR. However, the breakout does not yet take place, and the shell does not fragment. The model corroborates the statement of MLMCN that the MSRs are confined by the large scale height of the Fuchs and Thielheim (1979) density distribution (it should be noted that in terms of scale heights climbed by the shell Fig. 1a should be compared to Fig. 2b of MLMCN). A fully developed breakout can be observed in Fig. 1b which shows a Gaussian model with a cold halo at an age of $5.57 \times 10^{6} \mathrm{yr}$. In agreement with $\mathrm{MLMCN}$ it is found that only a small percentage of the shell mass is accelerated directly upwards, and that a secondary main shock wave sweeping through the halo forms, after the shell has fragmented. Interior to that wave 
the halo gas is rapidly mixed with the SN ejecta and the disk gas which diffused from the shell into the MSR. The secondary wave also sweeps through the upper layers of the disk and heats them up to several hundred thousands $K$.
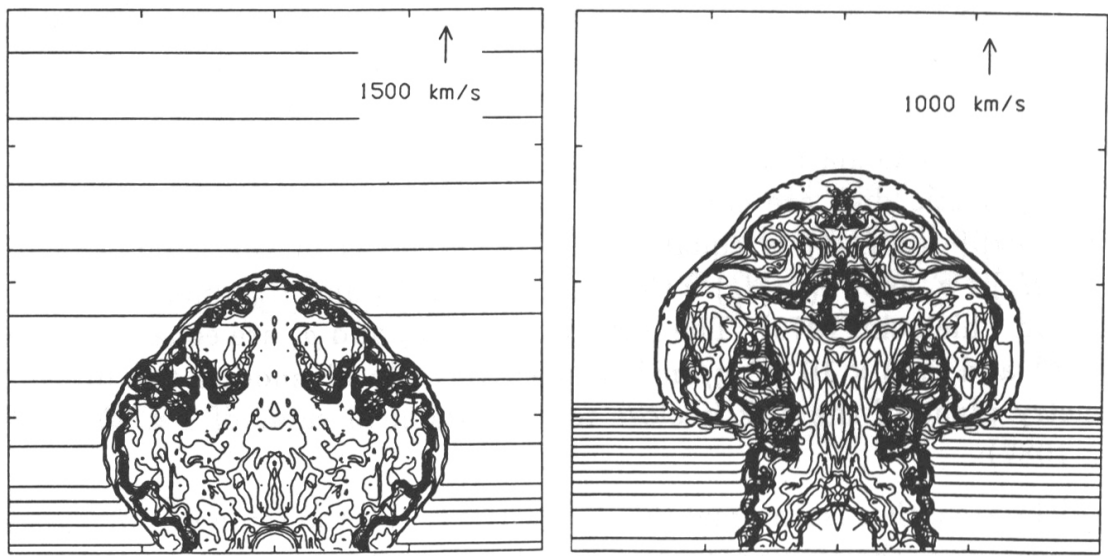

Fig.1. Density distributions and velocity fields of models $A$ and $B$ (frames a and b taken at an evolutionary age of $2.91 \times 10^{7}$ and $5.57 \times 10^{6} \mathrm{yr}$, respectively). The contours are logarithmically spaced with $\Delta \log (\rho)=0.2$. The distance betwen tick marks is $500 \mathrm{pc}$ in frames a, and $250 \mathrm{pc}$ in frame b.

\section{TABLE 1}

Parameters of the Models

\begin{tabular}{|c|c|c|c|c|c|c|c|}
\hline \multirow{2}{*}{ case } & \multicolumn{4}{|c|}{ disk } & halo & supernovae & \multirow{2}{*}{ remarks } \\
\hline & $P_{0}$ & $\rho_{0}$ & $\mathrm{~T}_{0}$ & $\operatorname{distr} \rho \operatorname{distr} \mathrm{T}$ & $\begin{array}{lll}P_{H} & \rho_{H} & \mathrm{~T}_{H}\end{array}$ & rate mass & \\
\hline $\mathbf{A}$ & $4_{10}^{-1}$ & & $0^{3}$ & composite unif & & std std & large grid \\
\hline $\mathbf{B}$ & $4_{10}^{-1}$ & & $0^{3}$ & Gaussian unif & $3_{10}^{-15} 5$ & std std & - \\
\hline C & $4_{10}^{-}$ & & $\sigma_{10}$ & composite unif & $4_{10}^{-14} 5_{10}^{-27} 5_{10}^{5}$ & std std & - \\
\hline D & $4_{10}^{-}$ & & $3_{10}{ }^{3}$ & composite unif & $4_{10}^{-14} 5_{10}^{-27} 5_{10}^{5}$ & low std & low sn rate \\
\hline $\mathbf{E}$ & $4_{10}^{-}$ & $17^{-2}$ & $3_{10} 0^{3}$ & Gaussian unif & $3_{10}^{-15} 5_{10}^{-27} 8_{10}$ & std high & low resolution \\
\hline F & $1_{10}^{-}$ & $17^{-2}$ & $8_{10}^{3}$ & Gaussian var & $7_{10}^{-13} 5_{10}^{-27} 1_{10}$ & std high & low resolution \\
\hline $\mathbf{G}$ & $1_{10}^{-}$ & $1.7_{10}^{-24}$ & $8_{10}^{3}$ & composite var & $7_{10}^{-13} 5_{10}^{-27} 1_{10}$ & std high & low resolution \\
\hline $\mathrm{H}$ & $1-$ & $1.7_{10}^{-24}$ & $8_{10}^{3}$ & composite var & $7_{10}^{-13} 5_{10}^{-27} 1_{10}$ & std high & low res evap \\
\hline
\end{tabular}

\section{Cases C-D.}

In these models a hot $\left(5 \times 10^{5} \mathrm{~K}\right)$ halo was placed atop the composite disk. Case $\mathrm{C}$ (Fig.2a) is shown at $1.32 \times 10^{7} \mathrm{yr}$, when the upper one-third of the MSR extends into the halo and drives a sound wave into it (the velocity of the shell has already fallen below the sound velocity of the hot halo gas, so that the propagation of a shock wave through the halo is inhibited). The diameter of the MSR measured in the disk plane amounts to about $500 \mathrm{pc}$. To reach a comparable size in Case D evolving at a low $\mathrm{SN}$ rate, $1.94 \times 10^{7} \mathrm{yr}$ are needed (Fig.2b). However, even at that time the internal instability is not so well developed as in Case $\mathrm{E}$ (in the latter case more wavelenghts seem to be excited and the average length of the dense tongues is larger; some of them 
have even separated from the shell). Both cases show once again that the growth rate of the internal instabilities depends on the way the energy is injected into the MSR. In both cases mixing of halo and ejecta is impossible. To enable it, the upper part of the shell would have to be either broken or washed out by the diffusion.

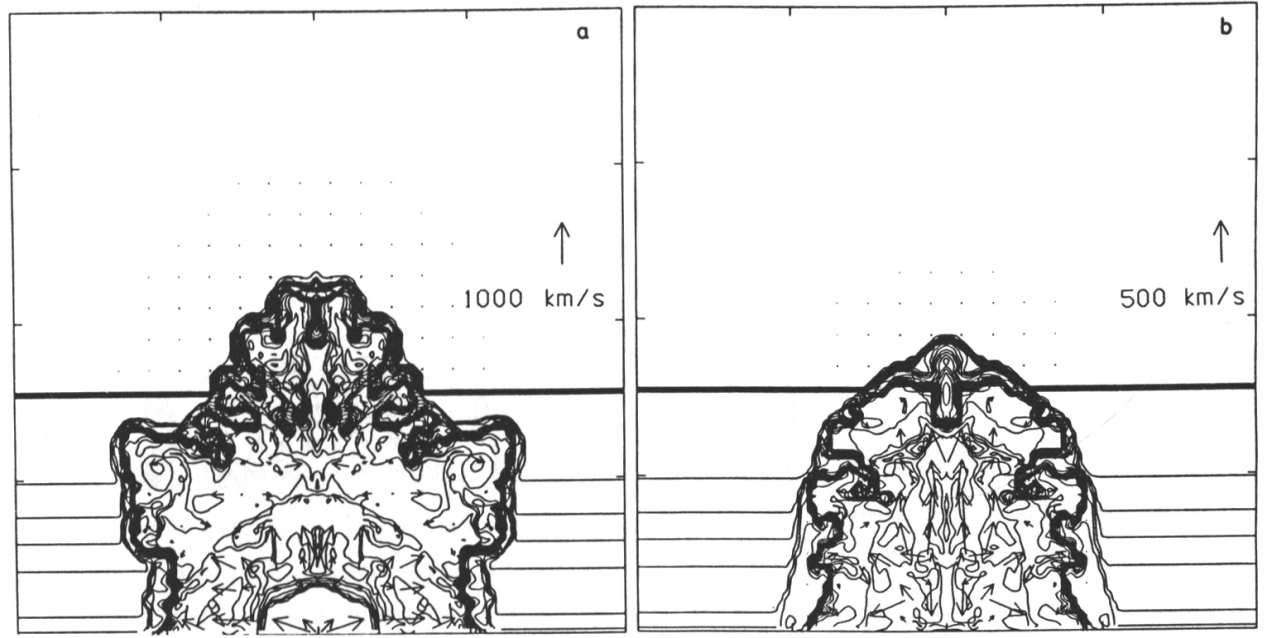

Fig.2. Density distributions and velocity fields of models $C$ and D (frames a and b taken at an evolutionary age of $1.32 \times 10^{7}$ and $1.94 \times 10^{7} \mathrm{yr}$, respectively). Contour spacing like in Fig.1. The distance between tick marks is $250 \mathrm{pc}$ in both frames.

\section{Cases E-F.}

These models illustrate the influence of the halo temperature on the late evolutionary stages of MSRs breaking out of a Gaussian disk, and are shown at approximately the same age of $1.2 \times 10^{7} \mathrm{yr}$ (Fig. 3). An extra mass is added to the SN ejecta in both models, and the resolution is decreased to $5 \mathrm{pc}$ (the amount of information lost due to poorer resolution is insignificant as far as the global properties of the models are considered). In Case $\mathrm{E}$ (cold halo, Fig. 3a) essentially the same phenomena as in Case $B$ are observed, the only difference being the size of the MSR. The flow within the MSR is very complicated: freely expanding ejecta are first shocked in an almost spherical primary wave, then they expand again in a nozzle formed by the broken shell to reach higly supersonic velocities, and finally they are re-shocked in an almost horizontal secondary wave. While spreading above the secondary wave, they are directed sideways and downwards, so that they reach the disk again.

In Case F (hot halo, Fig. 3b) the flow pattern is entirely different: instead of multiple shocks a gentle, sonic expansion prevails, directed predominantly upwards. Mixing between the interior gases and the halo mainly occurs in a series of vortices resembling puffs of smoke from a broad chimney. It should also be noticed that unlike in Case $\mathrm{E}$, the whole halo has already been set in motion (the motions of the halo are now induced by sound waves, whose speed is higher than the speed of the shock wave in Case $E$ due to the high temperature of the halo). Furthermore, the sound waves from the halo induce sound and weak shock waves in the upper layers of the disk. Typical velocities in a large vortex range from 100 to $200 \mathrm{~km} / \mathrm{s}$. Velocities in the halo far away from the vortices do not exceed $50 \mathrm{~km} / \mathrm{s}$, and those in the upper disk are smaler than $10 \mathrm{~km} / \mathrm{s}$. 


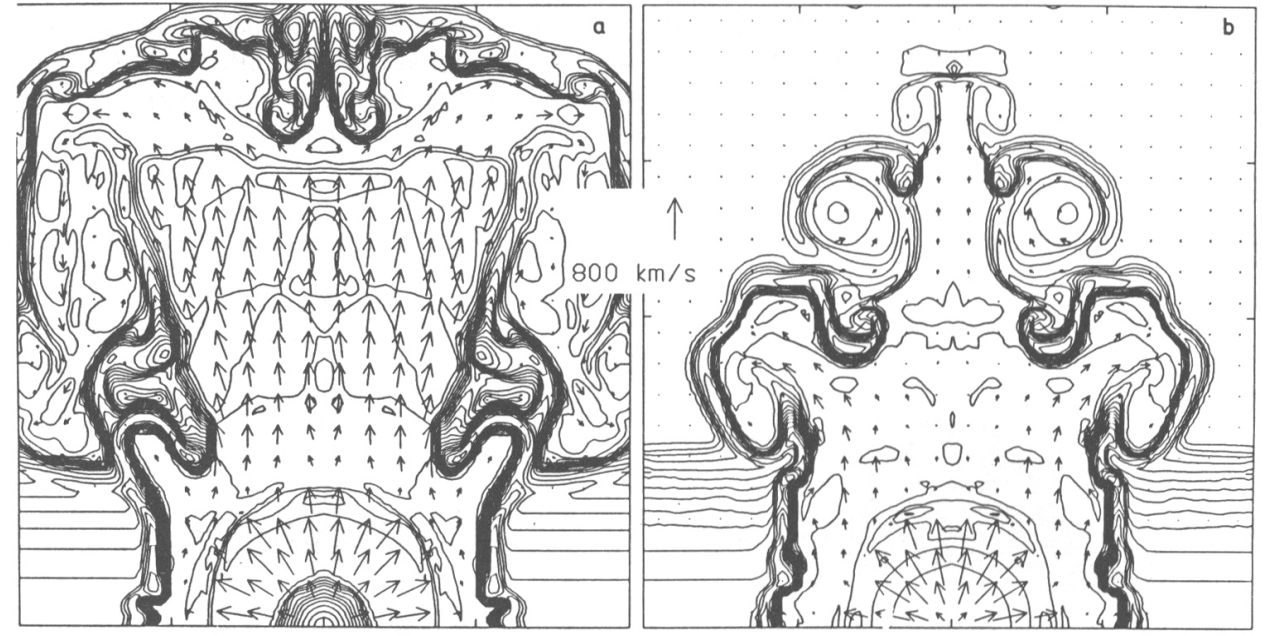

Fig.3. Density distributions and velocity fields of models $\mathrm{E}$ and $\mathrm{F}$ (frames a and b taken at an evolutionary age of $1.18 \times 10^{7}$ and $1.21 \times 10^{7} \mathrm{yr}$ respectively). Contour spacing like in Fig.1. The distance between tick marks is $230 \mathrm{pc}$ in both frames.

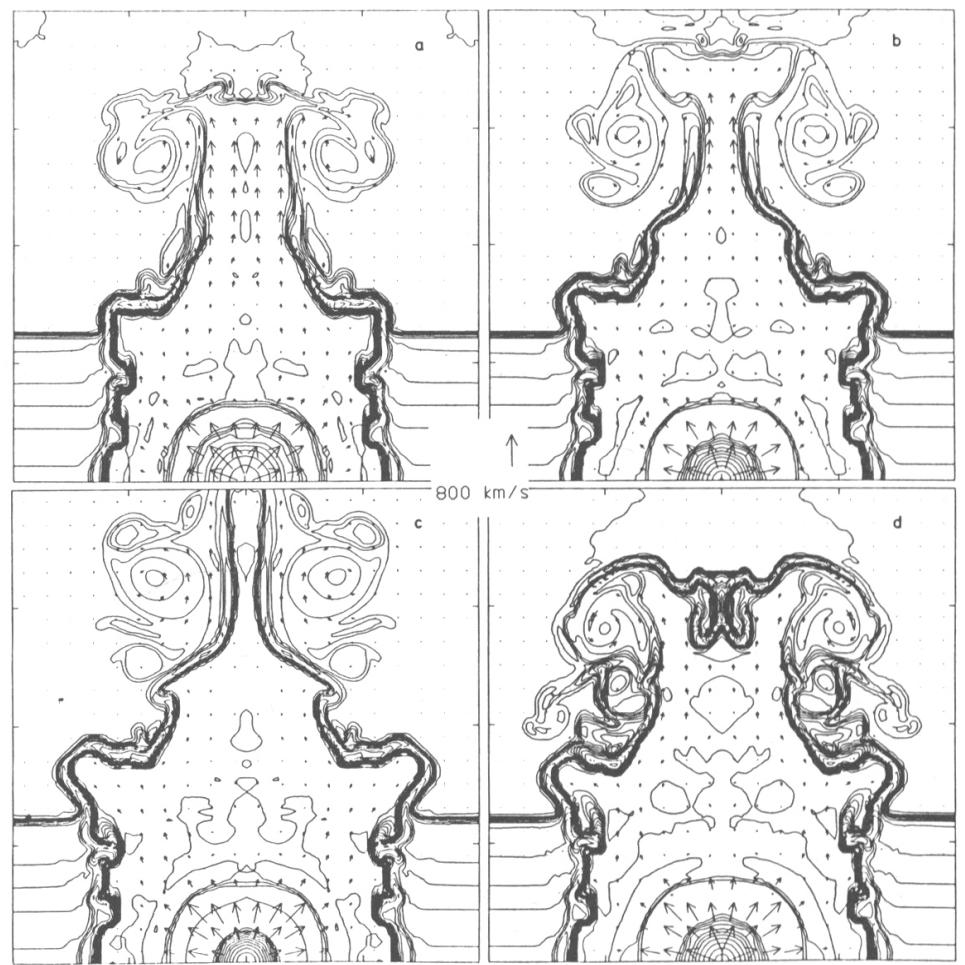

Fig.4. Density distributions and velocity fields of models $G$ (frames a,b and $c$ taken at an evolutionary age of $1.45 \times 10^{7}, 1.58 \times 10^{7}$ and $1.71 \times 10^{7}$ respecively), and $\mathrm{H}$ (shown at an evolutionary age of $1.72 \times 10^{7} \mathrm{yr}$ in frame d). Contour spacing like in Fig.1. The distance between tick marks is $230 \mathrm{pc}$ in all frames. 
In these calculations the late evolutionary stages of MSRs developing in a composite disk with a hot halo are examined. An extra mass is added to the SN ejecta in both models, and in Case $\mathrm{G}$ the mass is gradually removed from the shell on a time scale of $1 \times 10^{7} \mathrm{yr}$ (only the part of the shell extending into the halo is affected by this process). Both runs demonstrate the possibility of the breakout. The overall flow pattern is similar to the one observed in Case F: the whole halo is set in motion, and mixing proceeds in a number of vortices. The outflow from the interior of the MSR into the halo can be very efficient, and occasionally velocities up to $400 \mathrm{~km} / \mathrm{s}$ are reached in the "chimney". On the other hand, the vortices may occasionally squeeze the chimney and quench the outflow (shortly afterwards a new vortex begins to develop). The last models obtained in both cases may be compared in Figs. $4 \mathrm{c}$ and $4 \mathrm{~d}$ : in the latter figure the chimney is appreciably broader due to the inhibited squeezing action of the vortices which are hampered by dense fragments of the shell. The fragments of the shell in the halo move at a speed of $30 \mathrm{~km} / \mathrm{s}$.

\section{DISCUSSION.}

The calculations described in the present paper generally agree with the results of MLMCN and Iumentshchev et al. (1989). In particular, it is found that long-lived, fairly regular MSRs form in composite disks without halos (Case A). Such structures are able to reach radii of $1 \mathrm{kpc}$ and ages in excess of $3 \times 10^{7} \mathrm{yr}$ (still later evolutionary stages may be dominated by the gravity of the stellar component of the galactic disk which was not taken into account in the models). The SN ejecta are contained in them and cool rather in place than spread throughout the disk.

On the other hand, the MSRs break readily out of Gaussian disks with cold halos (Cases $B$ and E). Evolutionary phases preceding the breakout are very short $(t<3$ $\mathrm{Myr}$ ). The shell fragments upon breaking out, and releases the SN ejecta into the halo. However, a secondary shell composed of halo gas is formed immediately afterwards and direct mixing between the ejecta and the halo is inhibited (it is only possible due to the diffusion at the interface between the secondary shell and the ejecta). Compared to the composite case, a large fraction of the MSR energy goes into heating the halo rather than increasing the kinetic and potential energy of the shell. MSRs assume mushroom shapes and spread sideways above the disk, so that it is possible for them to merge and to form extended, hot regions $\left(T>10^{6} \mathrm{~K}\right)$ far above the symmetry plane of the disk. Thus, it is conceivable that even a thermally uniform galactic environment will evolve towards a thermally stratified one, with a hot halo atop of a rather cool disk.

In the presence of a hot halo, independently of the assumed stratification of the disk, chimneys are formed rather than mushrooms (Cases F-H). Due to sound waves excited at the outer edge of the shell during the breakout, and subsequently due to vortices above the already formed chimney, extended turbulent $r$ egions develop around every MSR. Excited primarily in the halo, the turbulence spreads into the upper layers of the disk via sound and/or weak, randomly oriented shock waves. Upon the breakout the shell is not screened from the halo by a shock wave, so that the diffusion may act on its both sides, leading to a rapid evaporation. As a result, shortly after the MSR has reached the base of the halo, an efficient dynamical mixing between the ejecta and the halo gas is enabled, and the metal-enriched gas may propagate to large distances from the center of the bubble, also permeating the upper disk.

The time scale for the formation of a chimney is of the order of 10-15 Myr, whereas the average lifetime of an $O B$ association is at least two times longer. To find the expected number of MSRs in a given sample of associations, however, an estimate of 
the lifetime of MSRs in the form of observable HI features is needed, which, due to the diffusion, may be rather short. Indeed, instabilities increasing the surface of the shell, and the fact that the diffusion operates on both sides of the shell, may bring the diffusion time scale down to a value much smaller than $\sim 100 \mathrm{Myr}$ characteristic of a bubble extending to $250 \mathrm{pc}$ in the Galactic plane (Mac Low and Mc Cray (1988). Since short wavelegth modes of the shell instabilities grow faster (Różyczka 1985 and references therein), a tenfold decrease of the diffusion time is entirely conceivable if one assumes that the rate of the diffusion from the shell is directly proportional the surface of the latter. In particular, the lifetime of an observable MSR might turn out to be shorter than the lifetime of its parent association. Unfortunately, the precise nature of the instabilities operating in the shell has not been revealed by the present calculations: as before, Rayleigh-Taylor and/or general thin shell effects may be in action. Very high resolution models of small regions of the shell would probably solve the problem.

The hot halo models provide a straightforward explanation to the missing coherent $\mathrm{HI}$ structures. They also suggest that qualitative corrections to the galactic circulation scenario proposed by Ikeuchi (1987) may be necessary, accentuating the importance of turbulent mixing. Moreover, it is likely that the circulation time (basically, the time the ejecta spend in the halo before getting incorporated into the disk again) may be much shorter than it was estimated on the basis of cooling rates (Heiles 1987, Ikeuchi 1987). Additional computational effort is needed to verify this hypothesis.

\section{ACKNOWLEDGMENTS.}

This work was partly supported by the grant CPBP 01.11 from the Polish Academy of Sciences.

\section{REFERENCES}

Bertschinger, E. 1986, Astrophys. J. 304, 154

Bloemen, J.B.M.G. 1987, Astrophys. J. 322, 694

Bruhweiler, F.C., Gull, T. Kafatos, M. and Sofia, S. 1980, Astrophys. J. Letters 238, L27

Cowie, L.L., Songaila, A., and York, D.G. 1979, Astrophys. J. 230, 469

Fuchs, B., and Thielheim, K.O. 1979, Astrophys. J. 227, 801

Heiles, C. 1984, Astrophys. J. Suppl. 55, 585

Heiles, C. 1987, Astrophys. J. 315, 555

Igumentshchev, I.V, Shustov, B.M., and Tutukov, A.V.: 1989, Astron.Astrophys. (submitted)

Ikeuchi, S. 1987, in Starbursts and Galaxy Evolution, ed. T.X. Thuan, T. Montmerle, J. Tran Thanh Van, p.27, Gif sur Yvette: Ed. Front

Lockman, F.J., Hobbs, L.M., Shull, J.M. 1986, Astrophys. J 301, 380

McCray, R. and Kafatos, M. 1987, Astrophys. J. 317, 190

Mac Low, M.-M. and McCray, R. 1988, Astrophys. J. 324, 776

Mac Low, M.-M., McCray, R., and Norman, M.L. 1989, Astrophys. J. 337, 141

Różyczka, M. 1985, Astron. Astrophys. 143, 59

Tenorio-Tagle, G. and Bodenheimer, P. 1988, Ann. Rev. Astron. Astrophys. 26, 145

Tenorio-Tagle, G., Bodenheimer, P., and Róźyczka, M. 1987, Astron. Astrophys. 182, 120

Tenorio-Tagle, G., Róźyczka, M., and Bodenheimer, P. 1989, Astron. Astrophys., submitted

Tomisaka, K., Habe, H., and Ikeuchi, S. 1981, Astrophys. Sp. Sci. 78, 273

Tomisaka, K., and Ikeuchi, S. 1986, Publ. Astr. Soc. Japan 38, 697

Umemura, S., Kazuo, I., and Kazunari, S. 1988, Publ. Astr. Soc. Japan 40, 25 


\section{DISCUSSION}

J.DYSON: What is the efficiency of conversion of the energy of a typical multiplesupernova into kinetic energy of the shell compared to the case of a single supernova in the ISM?

M.RÓZYYCZKA: As long as there is a continued energy input in the center of the MSR, and the nonspherical effects are negligible, the kinetic energy of the shell stays close to 0.1 of the total energy that has been delivered by supernovae. The largest conversion factors found for single explosion remnants amount to 0.08 , but in most cases they are by half an order of magnitude smaller. It is also known that until late evolutionary stages the radii of nonspherical MSRs, as measured in the symmetry plane of the galactic disk, grow proportionally to $t^{\beta}$, where $\beta$ is appreciably larger than the snowplough value of 0.25 (even in the early breakout $\beta \sim 0.44$ ). Thus, the MSRs which have not yet "discharged into the halo" convert the supernova energy into the kinetic energy of the shell more efficiently than their classical counterparts.

J.WAMPLER: Is the neglection of rotational effects in your models justified?

M.RÓŻYCZKA: At the solar circle the galactic shear does not cause appreciable effects for the first $30 \mathrm{Myr}$ of the MSR's evolution (at that time the MSR's cross-section in the equatorial plane becomes an ellipse whose axis ratio is rougly $2 / 3$ ). 